\title{
High levels of serum mannose-binding lectin are associated with the severity of clinical signs of leptospirosis
}

\author{
K.A. Miranda1, L.R.S. Vasconcelos ${ }^{1}$, L.C.B.B. Coelho², J.L. Lima Filho², \\ M.S.M. Cavalcanti ${ }^{1}$ and P. Moura ${ }^{1}$ \\ ${ }^{1}$ Instituto de Ciências Biológicas, Universidade de Pernambuco, Recife, PE, Brasil \\ ${ }^{2}$ Centro de Ciências Biológicas, Universidade Federal de Pernambuco, Recife, PE, Brasil
}

Correspondence to: P. Moura, ICB-UPE, Rua Arnóbio Marques, 310, 50100-130 Recife, PE, Brasil

Fax:+55-81-3421-1769. E-mail: patmoura@pq.cnpq.br

\begin{abstract}
The clinical heterogeneity observed in leptospirosis may be associated with host factors or bacteria virulence. Human serum mannose-binding lectin (MBL) recognizes many pathogens, and low levels of this lectin are associated with susceptibility to infection. $M B L$ is also implicated in the modulation of the inflammatory process. We determined the levels of serum $M B L$ during leptospirosis infection. A double-antibody sandwich ELISA was used to detect the immunoreactive serum MBL. The ELISA plates were coated with monoclonal antibody to $\mathrm{MBL}$ and bound $\mathrm{MBL}$ or recombinant human $\mathrm{MBL}$ were detected by rabbit antihuman MBL serum. HRPO-conjugated goat anti-rabbit antibody was used for detection of the reaction. Two groups of patients seen at referral hospitals in Recife, PE, Brazil, were divided according to the year of infection, $2001(\mathrm{~N}=61)$ or $2002(\mathrm{~N}=57)$ and compared in terms of disease severity and levels of serum MBL. A group of healthy volunteers $(N=97)$ matched by age, gender, and ethnic background was used as control. Patients infected in 2001 had more severe outcomes than those infected in 2002, including jaundice, hemorrhage, respiratory alteration, and renal complication $(P=0.0009$; chi-square test). The frequency of patients producing serum $M B L>1000 \mathrm{ng} / \mathrm{mL}$ was higher in the 2001 group than in the 2002 and control groups ( $P$ $<0.01$ ), suggesting an association of $M B L$ level with disease severity. The involvement of $M B L$ and genetic variation of the $M B L 2$ gene should be further evaluated to establish the role of this lectin in the pathogenesis of leptospirosis.
\end{abstract}

Key words: Leptospirosis; Mannose-binding lectin; Hemorrhage; Cardiac alteration; Renal complication

Received May 10, 2008. Accepted February 26, 2009

\section{Introduction}

The spectrum of leptospirosis signs or symptoms ranges from mild flu-like onset to a fatal form with multiorgan involvement including hepato-renal failure, myocarditis, severe pulmonary hemorrhage with respiratory distress, and meningitis (1). The major pathogenic mechanism of leptospirosis affecting multiple organs is diffuse capillarity $(1,2)$. Even though the virulence factors of leptospires are only partially known, the pathogenic specimens must have the ability to multiply in the blood, adhere to the endothelium and avoid the host's innate immune response, i.e., complement activation, antimicrobial peptide cytolysis and phagocytosis $(3,4)$. Non-pathogenic leptospires are killed by innate immunity mechanisms, including phagocytosis and complement activation $(1,4)$.

Complement system activation by innate immunity involves the recognition of pathogens by two distinct pathways, alternative and lectin (5). Patient isolates of Leptospira have been shown to have different degrees of resistance to the human complement system, while nonpathogenic strains isolated from water sources were sensitive (4). To escape complement, many pathogens use the deposition of complement inhibition molecules, such as factor $\mathrm{H}$, on their membranes. Verma et al. (6) described a Leptospira interrogans molecule that can bind to factor $\mathrm{H}$ (LfhA) avoiding complement activation. In addition, it was shown that human serum opsonization of pathogenic leptospires increased the adherence to neutrophils via the complement component C3 receptor (CR3) instead of 
facilitating phagocytic uptake (7).

The activation of the complement system's lectin pathway is mainly elicited by the mannose-binding lectin (MBL), which is a lectin type $C$ of innate immunity that binds to mannan present on the surface of microorganisms and mediates opsonophagocytosis directly or by the activation of the lectin pathway $(5,8)$. MBL levels in humans vary according to the polymorphism of the structural region (exon 1) of the MBL2 gene and/or the promoter (5).

The purification of genus-specific antigens from nonpathogenic L. biflexa has shown a common backbone structure that has mannan as its main component, which cross-reacts with serum elicited by pathogenic strains (9). Since MBL is the major molecule of the complement system's lectin pathway acting before pre-existing antibodies, it could be an important molecule in innate immunity interacting with the leptospires during infection.

Studies have shown that MBL can modulate the inflammation process as well as enhance activation of the complement cascade (10). Therefore, the correlation of MBL and clinical symptoms for leptospirosis could be related to the immunomodulatory role of this lectin, which thus may contribute to the outcome of different clinical manifestations.

Serum levels of MBL have not been investigated during human or animal model leptospirosis infection. Thus, the objective of this study was to determine if there is an association of the severity of leptospirosis with MBL levels.

\section{Material and Methods}

Sera from patients seen at the Oswaldo Cruz University Hospital, University of Pernambuco (HUOC-UPE), which is a referral hospital for leptospirosis in Recife, PE, Brazil, were obtained from the Epidemiological Unit of the Central Laboratory of Pernambuco (LACEN-PE). Data from leptospirosis cases in the years $2001(\mathrm{~N}=61)$ and $2002(\mathrm{~N}$ $=57$ ) were compared regarding the outcome of the onset of disease. Severity was assessed in terms of the occurrence of the most important clinical signs: jaundice, hemorrhage, conjunctive congestion, respiratory alteration, meningisms, diarrhea, cardiac alteration, and renal complication.

To confirm the cases of leptospirosis, LACEN-PE used commercial enzyme-linked immunosorbent assays (ELISA) from Biomanguinhos (Rio de Janeiro, RJ, Brazil). Microagglutination tests were also performed to test positive sera to identify serovar; however, these data were not available to us.

Samples from patients that were sent to LACEN-PE and could be recovered were identified with a registration number for the laboratory where they were processed, so they could not be matched individually to the HUOC-UPE patients' charts. Therefore, the cases were analyzed as a whole; mean age was 25 and 23 years in the 2001 and 2002 groups, respectively. There were 10 females and 51 males in the 2001 group and 11 females and 46 males in the 2002 group. Ninety-seven healthy volunteers were matched with patients according to gender, age, and ethnic background, and those without clinical history of renal insufficiency, thyroid disease or diabetes participated as part of the control group, yielding a total of 33 females and 64 males, with a mean age of 32 years. The project was approved by the HUOC-UPE Bioethics Scientific Committee (No. 31.05.0046) and authorization to assess the serum samples of leptospirosis patients was obtained from LACEN-PE. The healthy control volunteers gave written informed consent.

Recombinant human MBL and monoclonal antibody (mAb-6), as well as polyclonal rabbit antiserum against human MBL, were kindly provided by Drs. Kazue Takahashi and Alan Ezekowitz (Department of Pediatrics, Massachusetts General Hospital, Boston, MA, USA). HRPO-conjugated goat immunoglobulin was purchased from Sigma (USA).

ELISA was used to measure MBL in serum samples. A double-antibody sandwich ELISA detected the total immunoreactive $\mathrm{MBL}$ in the sample. The wells of a microtiter plate (Nunc-Maxisorp, Denmark) were coated overnight with $50 \mu \mathrm{L}$ anti-human MBL mAb-6 IgG $(5 \mu \mathrm{g} / \mathrm{mL}$ in PBS, $\mathrm{pH}$ 7.4), blocked with $10 \mathrm{mg} / \mathrm{mL} B S A$ for $1 \mathrm{~h}$, and then washed with PBS containing $0.05 \%$ Tween-20 (PBST). Serum samples were diluted $1 / 100$ in PBST containing 5 $\mathrm{mg} / \mathrm{mL}$ BSA (BSA5-PBST), and $50 \mu \mathrm{L}$ was added in duplicate to the mAb-6-coated wells and incubated overnight at $4^{\circ} \mathrm{C}$. After washing, bound MBL was detected by incubation with $50 \mu \mathrm{L}$ rabbit anti-human MBL serum (1/300 in BSA5-PBST) for $3 \mathrm{~h}$. After further washing, $50 \mu \mathrm{L} /$ well HRPO-conjugated goat anti-rabbit immunoglobulin was added and incubated for $1 \mathrm{~h}$ at room temperature. Following further washing, $100 \mu \mathrm{L}$ of substrate $0.2 \mathrm{mM}$ ABTS [2,2'-azino-bis(3-ethylbenzthiazoline-6-sulfonic acid)] in 50 $\mathrm{mM}$ citrate buffer, $\mathrm{pH} 4.0$, containing $0.004 \% \mathrm{H}_{2} \mathrm{O}_{2}$ was added. Color development was stopped after $20 \mathrm{~min}$ by the addition of $50 \mu \mathrm{L} \mathrm{NaF}(192 \mathrm{mg} / \mathrm{mL})$, and absorbance was read at dual wavelengths of $405 / 450 \mathrm{~nm}$. Based on the range of human serum $M B L$ levels described in the literature (11), three groups were created as follows: low (0 to $<500 \mathrm{ng} / \mathrm{mL})$, intermediate $(500-1000 \mathrm{ng} / \mathrm{mL})$, and high $(>1000 \mathrm{ng} / \mathrm{mL})$. Statistical analysis for the difference in frequency was performed using the Statistica software version 6.0 (USA). P values $<0.05$ were regarded as 
Table 1. Clinical outcome of leptospirosis patients who had been infected during 2001 or 2002.

\begin{tabular}{lcc}
\hline Clinical outcome & $2001(\mathrm{~N}=61)$ & $2002(\mathrm{~N}=57)$ \\
\hline Jaundice $^{*}$ & $56(91.8 \%)$ & $36(63.1 \%)$ \\
Hemorrhage* $^{*}$ Conjunctive congestion $^{*}$ & $41(67.2 \%)$ & $9(15.7 \%)$ \\
Respiratory alteration* & $42(60.3 \%)$ & $14(24.5 \%)$ \\
Meningisms & $42(68.8 \%)$ & $7(12.2 \%)$ \\
Diarrhea & $45(73.4 \%)$ & $2(3.5 \%)$ \\
Cardiac alteration $_{\text {Renal complication* }}^{*}$ & $41(67.2 \%)$ & $1(1.7 \%)$ \\
& $50(81.9 \%)$ & $10(17.5 \%)$ \\
\hline
\end{tabular}

Data are reported as number of patients with percent within parentheses. Patients had at least one episode of clinical symptoms. ${ }^{*} \mathrm{P}=0.0009$ (chi-square test).
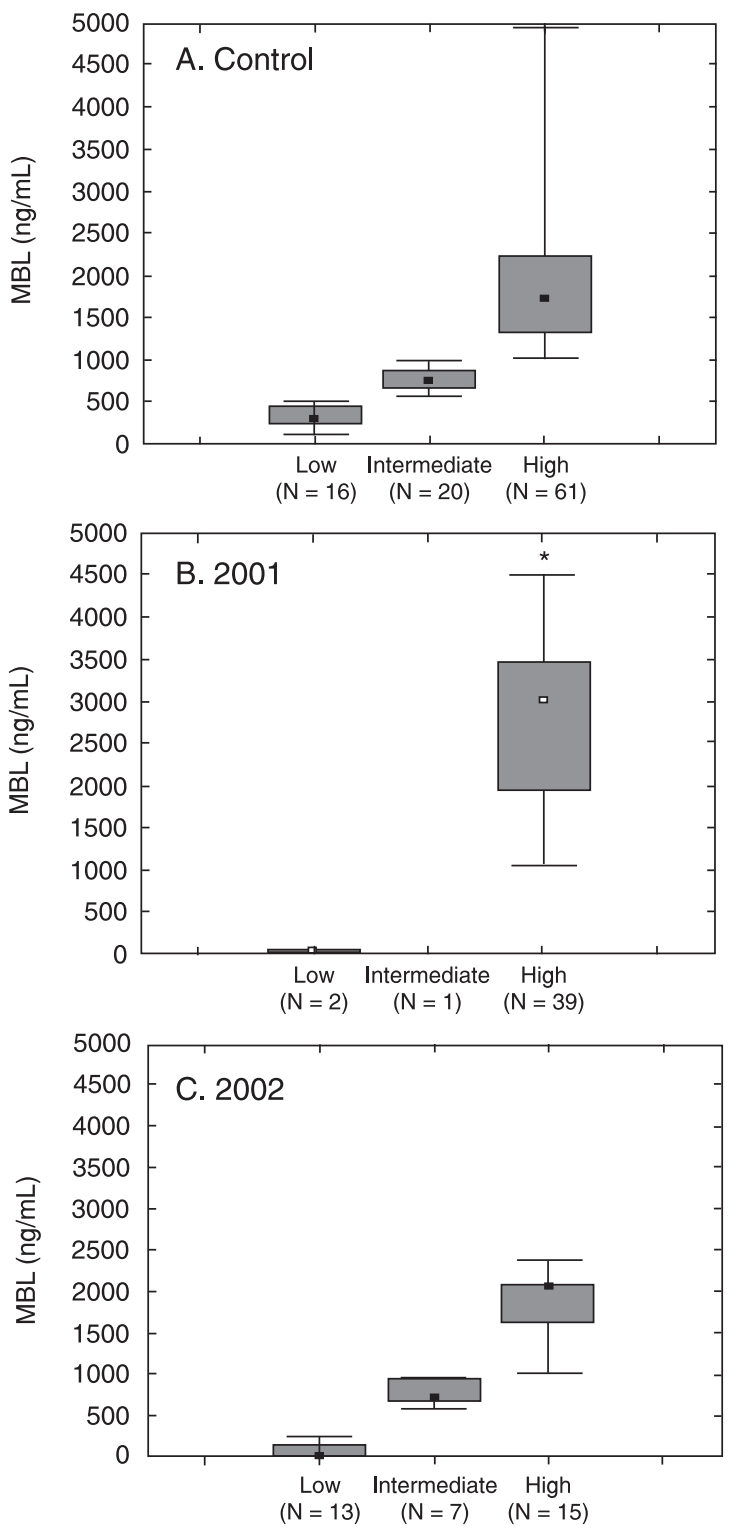

statistically significant. Frequencies were compared by the chi-square test, and MBL concentrations were compared by the Mann-Whitney test (two-tailed).

\section{Results}

The analysis of the 2001 and 2002 groups showed similarity regarding gender and age, with a predominance of male cases. The range in age of those most affected was 16 to 20 years, with all epidemiological aspects in accordance with the current literature (2). Nevertheless, the severity indicators were clearly distinct. In the 2001 group, $13 \%$ of the cases were fatal, while the fatality rate of the 2002 group was $3.5 \%$. The clinical signs indicating severity are reported in Table 1. Jaundice, hemorrhage, respiratory alteration, and renal complication, which are related to the severe form of leptospirosis, Weil's disease syndrome, were represented in a higher proportion in the 2001 group than in the 2002 group ( $P=0.0009$; chi-square test).

Of the 61 cases in the 2001 group, it was possible to recover 42 sera, and 35 sera were recovered from the 57 cases of the 2002 group. The determination of serum MBL was performed on $68.9 \%$ (2001) and 61.4\% (2002) of patients. This fact probably did not affect the association of MBL serum levels with clinical signs observed in the groups; the loss of samples was random and similar for each group. The MBL median (range) was $1229 \mathrm{ng} / \mathrm{mL}$ (1214947), $2935 \mathrm{ng} / \mathrm{mL}$ (40-4472), and $776 \mathrm{ng} / \mathrm{mL}$ (40-3051) for the control, 2001 and 2002 groups, respectively. The median MBL was significantly elevated in patients of the 2001 group compared with controls or patients of the 2002 group $(P<0.01)$. The MBL median of the 2002 group was lower compared to controls $(P=0.02)$.

The patients of the 2001 group were characterized by an elevated frequency of high (39/42) levels of MBL (Figure 1B), while the 2002 group showed a similar distribution of high (7/35), intermediate (13/35), and low (9/35) levels of $\mathrm{MBL}$ (Figure 1C). The patients with $\mathrm{MBL}>1000 \mathrm{ng} / \mathrm{mL}$ were more frequent in the 2001 group compared with those in the 2002 or control groups $(P<0.0001$ for 2001 vs

Figure 1. Mannose-binding lectin (MBL) levels of control and patient groups diagnosed with leptospirosis in the years 2001 and 2002. Distribution of MBL concentrations in controls $(A)$, in patients with leptospirosis diagnosed in 2001 (B) and diagnosed in 2002 (C) was considered to be low ( 0 to $<500 \mathrm{ng} / \mathrm{mL}$ ), intermediate $(500-1000 \mathrm{ng} / \mathrm{mL})$, or high $(>1000 \mathrm{ng} / \mathrm{mL})$. The box plot indicates $25-75 \%$ variance, the top and bottom horizontal bars represent the minimum and maximum, and the small square within the box plot represents the median. Elevated MBL levels were more frequent in patients from 2001 vs control and 2001 vs 2002 patients $(P<0.001$, chi-square test). 
2002 and $P=0.0012$ for control vs 2001).

\section{Discussion}

The MBL concentration obtained for the control group in this study was similar to those reported by other studies of the Brazilian population $(12,13)$. The frequency of $\mathrm{MBL}$ levels $<100 \mathrm{ng} / \mathrm{mL}$ in the control group was, however, very low $(2 \%)$ compared to the genotypic frequency of $\mathrm{MBL}$ polymorphisms $(6 \%)$ reported for a group of individuals with the same ethnic background (14). This may be explained by the fact that the monoclonal antibody used in the present study recognized both high- and low-molecular weight variants of $M B L$, making the amount of $M B L$ in circulation less dependent on the presence of structural variant alleles detected by genotyping $(11,14)$.

Yet, direct comparisons between genetic studies of $\mathrm{MBL}$ and the amount of serum MBL reported here are difficult to make. Although, the differences observed in the levels of MBL between the two groups could be associated with genetic polymorphisms of MBL, the patient's basal levels of MBL could not be measured, and this aspect could not be further investigated. Studies investigating the genotyping of $M B L 2$ are, therefore, encouraged to provide evidence of an association between polymorphisms for MBL levels and the severity of symptoms for leptospirosis.

The heteroligomeric forms of $\mathrm{MBL}(\mathrm{A} / 0)$ have shown a low amount of complement deposition activity, which raise the question of their physiological role in the serum $(5,11)$. The immunoreactive ELISA utilized in the present study suggests a new approach to the determination of high- and low-molecular weight forms of circulating MBL with a monoclonal antibody, while the commercial kits for MBL determination recognize only the high-molecular weight forms. Since our population has shown a high frequency of heterozygous individuals (30\%) for MBL (14), the monoclonal ELISA employed had the advantage that it could detect either low- or high-molecular weight forms of MBL.

Low levels of MBL have been linked to susceptibility to infectious disease. Nonetheless, low serum MBL has been associated with protection from the development of a lepromatous form of Hansen's disease (12). It was suggested that Mycobacterium leprae uses the deposition of opsonins produced by the complement system to enter the host cell. This also suggests that low levels of MBL represent a protection factor since they may be associated with minimal production of inflammatory components, which is a main complication of leptospirosis.

The function of MBL in inflammation is multifaceted, and its mechanisms of action is poorly understood. It is believed that $\mathrm{MBL}$ is able to induce monocyte release of proinflamma- tory cytokines, such as TNF- $\alpha$ (10). In leptospirosis, the ratio of IL-10 to TNF- $\alpha$ is associated with disease progression and a high ratio of IL-10/TNF- $\alpha$ predicts a good prognosis (15). The expression levels of proinflammatory and antiinflammatory cytokines were investigated in a hamster model infected with L. interrogans (16). In this study, the authors showed early pronounced expression of Th1 cytokine mRNAs, i.e., TNF- $\alpha$ and INF- $\gamma$ after infection, while expression of IL-4 and IL-10, which are anti-inflammatory cytokines, was observed 2-3 days post-infection. The variation in median levels of MBL in the leptospirosis patients from 2001 and 2002 differed by more than a factor of 3 (2935 and 776 $\mathrm{ng} / \mathrm{mL}$, respectively), which could influence the balance of IL-10/TNF- $\alpha$ during the course of disease.

Dorigatti et al. (17), using glycoprotein of pathogenic $L$. interrogans serovar Copenhagen from a patient with Weil's syndrome, demonstrated the induction of IL-6 production and activation of monocytes by this component. Thus, the elevated MBL levels found in the patients from 2001 could be related to the inflammatory response and the presence of Leptospira virulence factors such as glycoprotein of pathogenic Leptospira (17). The lower levels of MBL found in the 2002 group suggest that the inflammatory response to infection was milder due to an effective immune response or the lack of Leptospira virulence factors in most cases. Unfortunately, it was not possible to isolate the leptospires from these patients to confirm this hypothesis.

In addition to the immune response of the host, the lethality of leptospiral infection has been attributed to changes in lipopolysaccharide $\mathrm{O}$-antigen, which has rhamnose as its major component and which represents a potential binding site for MBL $(1,3,9)$. The variability of Leptospira glycoproteins and antigens associated with lipopolysaccharide may be implicated in the differential binding ability of MBL, and, thus, its inflammatory modulation potential by complement activation and monocyte cytokine production (10,17-19).

We observed that the high frequency of patients presenting MBL levels >1000 ng/mL was associated with a higher percentage of cardiac alterations, hemorrhage, respiratory distress, and renal complication. Troelsen et al. (20) reported that patients with rheumatoid arthritis, who presented serum MBL higher than $3000 \mathrm{ng} / \mathrm{mL}$, had a significant risk of ischemic heart disease and myocardial infarction.

Our results suggest that serum MBL may be the link between the humoral innate immune response and the differential spectrum of severity observed in infected individuals. Nevertheless, as an immunomodulator, MBL may be protective or may aggravate the illness depending on its level, physicochemical status, microenvironment, and cytokine profile induced by leptospires antigen variations. 


\section{Acknowledgments}

We thank Dr. E. Margot Anders for her essential help with the establishment of the immunoreactive MBL ELISA protocol and the Epidemiological Unit of the Central Laboratory of Pernambuco (LACEN-PE) for the sera samples of the patients with leptospirosis.

\section{References}

1. Bharti AR, Nally JE, Ricaldi JN, Matthias MA, Diaz MM, Lovett MA, et al. Leptospirosis: a zoonotic disease of global importance. Lancet Infect Dis 2003; 3: 757-771.

2. Levett PN. Leptospirosis. Clin Microbiol Rev 2001; 14: 296326.

3. Nally JE, Chow E, Fishbein MC, Blanco DR, Lovett MA. Changes in lipopolysaccharide $\mathrm{O}$ antigen distinguish acute versus chronic Leptospira interrogans infections. Infect Immun 2005; 73: 3251-3260.

4. Meri T, Murgia R, Stefanel P, Meri S, Cinco M. Regulation of complement activation at the C3-level by serum resistant leptospires. Microb Pathog 2005; 39: 139-147.

5. Dean MM, Heatley S, Minchinton RM. Heteroligomeric forms of codon 54 mannose binding lectin (MBL) in circulation demonstrate reduced in vitro function. Mol Immunol 2006; 43: 950-961.

6. Verma A, Hellwage J, Artiushin S, Zipfel PF, Kraiczy P, Timoney JF, et al. LfhA, a novel factor $\mathrm{H}$-binding protein of Leptospira interrogans. Infect Immun 2006; 74: 2659-2666.

7. Cinco M, Cini B, Perticarari S, Presani G. Leptospira interrogans binds to the CR3 receptor on mammalian cells. Microb Pathog 2002; 33: 299-305.

8. Neth O, Jack DL, Dodds AW, Holzel H, Klein NJ, Turner MW. Mannose-binding lectin binds to a range of clinically relevant microorganisms and promotes complement deposition. Infect Immun 2000; 68: 688-693.

9. Matsuo K, Isogai E, Araki Y. Utilization of exocellular mannan from Rhodotorula glutinis as an immunoreactive antigen in diagnosis of leptospirosis. J Clin Microbiol 2000; 38: 3750-3754.

10. Jack DL, Read RC, Tenner AJ, Frosch M, Turner MW, Klein NJ. Mannose-binding lectin regulates the inflammatory response of human professional phagocytes to Neisseria meningitidis serogroup B. J Infect Dis 2001; 184: 1152-1162.

11. Garred P, Larsen F, Madsen HO, Koch C. Mannose-binding lectin deficiency - revisited. Mol Immunol 2003; 40: 73-84.

12. Dornelles LN, Pereira-Ferrari L, Messias-Reason I. Man- nan-binding lectin plasma levels in leprosy: deficiency confers protection against the lepromatous but not the tuberculoid forms. Clin Exp Immunol 2006; 145: 463-468.

13. Schafranski MD, Stier A, Nisihara R, Messias-Reason IJ. Significantly increased levels of mannose-binding lectin $(\mathrm{MBL})$ in rheumatic heart disease: a beneficial role for MBL deficiency. Clin Exp Immunol 2004; 138: 521-525.

14. Segat L, Silva Vasconcelos LR, Montenegro de Melo F, Santos SB, Arraes LC, Moura P, et al. Association of polymorphisms in the first exon of mannose binding lectin gene (MBL2) in Brazilian patients with HCV infection. Clin Immunol 2007; 124: 13-17.

15. Tajiki H, Salomao R. Association of plasma levels of tumor necrosis factor alpha with severity of disease and mortality among patients with leptospirosis. Clin Infect Dis 1996; 23: 1177-1178

16. Vernel-Pauillac F, Merien F. Proinflammatory and immunomodulatory cytokine mRNA time course profiles in hamsters infected with a virulent variant of Leptospira interrogans. Infect Immun 2006; 74: 4172-4179.

17. Dorigatti $F$, Brunialti MK, Romero EC, Kallas EG, Salomao R. Leptospira interrogans activation of peripheral blood monocyte glycolipoprotein demonstrated in whole blood by the release of IL-6. Braz J Med Biol Res 2005; 38: 909-914.

18. Diament D, Brunialti MK, Romero EC, Kallas EG, Salomao R. Peripheral blood mononuclear cell activation induced by Leptospira interrogans glycolipoprotein. Infect Immun 2002; 70: $1677-1683$.

19. Abdulkader RC, Daher EF, Camargo ED, Spinosa C, da Silva MV. Leptospirosis severity may be associated with the intensity of humoral immune response. Rev Inst Med Trop São Paulo 2002; 44: 79-83.

20. Troelsen LN, Garred P, Madsen HO, Jacobsen S. Genetically determined high serum levels of mannose-binding lectin and agalactosyl IgG are associated with ischemic heart disease in rheumatoid arthritis. Arthritis Rheum 2007; 56: 21-29. 